This item was submitted to Loughborough's Research Repository by the author.

Items in Figshare are protected by copyright, with all rights reserved, unless otherwise indicated.

\title{
Laser vibrometry on solid surfaces : the effects of laser speckle
}

PLEASE CITE THE PUBLISHED VERSION

PUBLISHER

(C) Laser Institute of America

VERSION

AM (Accepted Manuscript)

LICENCE

CC BY-NC-ND 4.0

REPOSITORY RECORD

Rothberg, Steve, and Neil A. Halliwell. 2019. "Laser Vibrometry on Solid Surfaces : The Effects of Laser Speckle”. figshare. https://hdl.handle.net/2134/9650. 
This item was submitted to Loughborough's Institutional Repository (https://dspace.lboro.ac.uk/) by the author and is made available under the following Creative Commons Licence conditions.

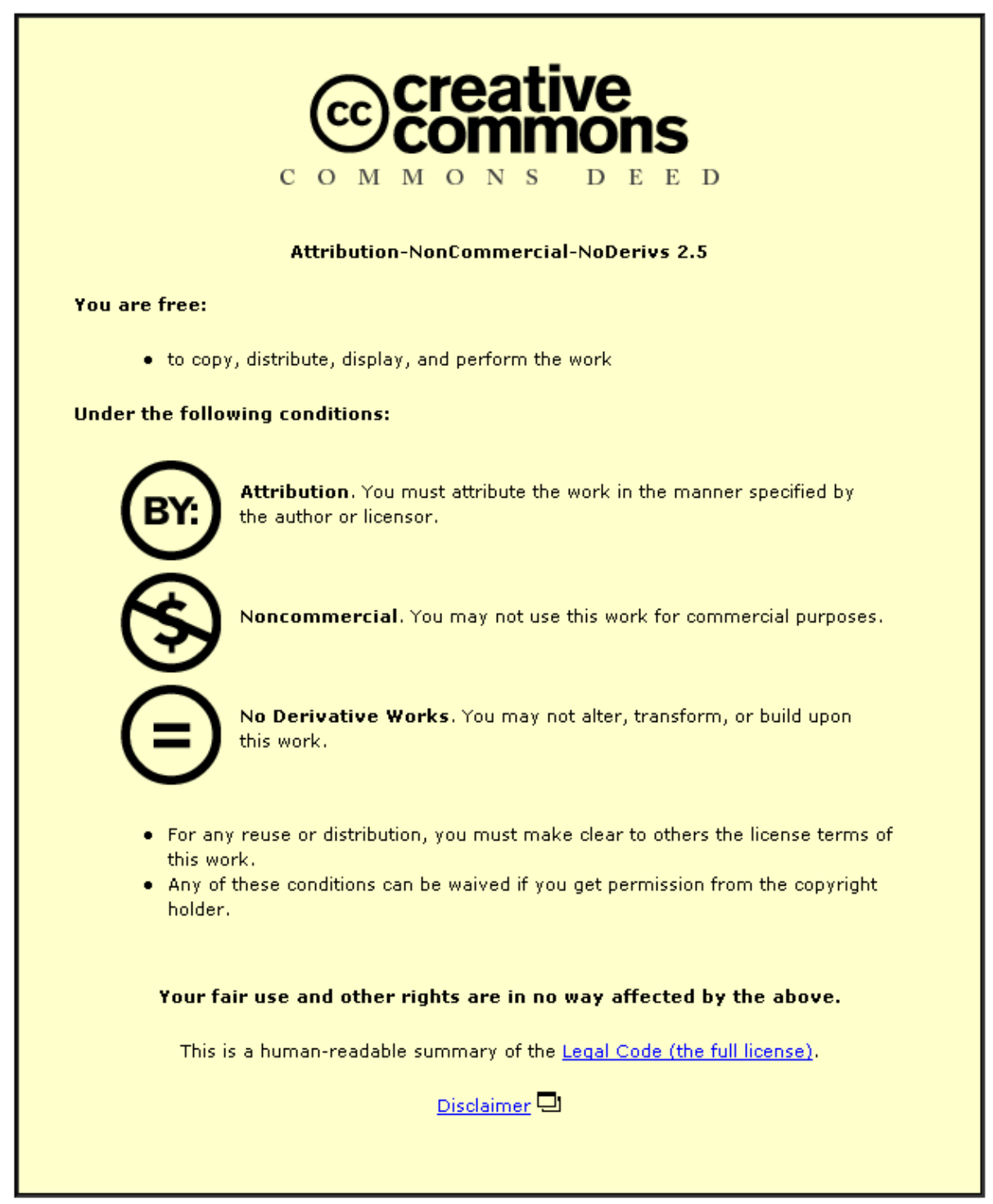

For the full text of this licence, please go to: http://creativecommons.org/licenses/by-nc-nd/2.5/ 
by

S.J.ROTHBERG and N.A.HALLIWELL

Department of Mechanical Engineering

Loughborough University

Loughborough

Leicestershire LE11 3TU

ENGLAND 


\section{ABSTRACT}

This letter discusses the phase and amplitude modulation of Doppler signals generated in laser Doppler velocimetry (LDV) systems with reference to the paper entitled "Dynamic Laser Doppler Velocimetry on Solid Surfaces" by Rajadhyaksha and Stevenson (Vol 4 No 2). In particular, transit time and velocity gradient frequency broadenings are described in terms of the speckle pattern behaviour on the photodetector and this approach is reconciled with the scattering element approach adopted by Rajadhyaksha and Stevenson. Furthermore, it is described how the frequency components of the Doppler phase noise can coincide with the vibration components of interest, thereby introducing ambiguity in interpretation of data. As a consequence, the reduction of Doppler phase noise by averaging is not generally acceptable. It is concluded that consideration of the dynamic speckle pattern is more appropriate to the investigation of Doppler phase noise since the speckle pattern itself has the ultimate influence on the LDV performance. 
INTRODUCTION

This letter refers to the paper entitled "Dynamic Laser Doppler Velocimetry on Solid Surfaces" by Rajadhyaksha and Stevenson (Vol 4 No 2 ) within which two points are felt to require further comment. The first concerns the distinction made in the paper between the effects of a target surface with "multiple scattering elements" and laser speckle. The second concerns the process of averaging over many Doppler cycles in order to increase the system accuracy.

The Rajadhyaksha and Stevenson system consisted of a HeNe laser amplitude divided by a beamsplitter and arranged to give two parallel laser beams. The beams were then brought together by a lens onto the face of a rotating roller. Scattered light was then focused by a second lens onto a photodetector whose output was processed to give a signal proportional to target velocity. The optical system is shown in figure 1.

Speckle Effects and Multiple Scattering Elements

Equation (1) of their paper presents an equation for the Doppler signal, $s(t)$, from a Laser Doppler Velocimetry (LDV) system as follows:

$s(t)=H(t) \cos \left[2 \pi f_{D} t-\phi(t)\right]$

where $t$ denotes time dependence, $H(t)$ is a random amplitude modulation function, $f_{D}$ is the expected Doppler frequency and $\phi(t)$ is a stochastic phase term. It is stated in the text of the Rajadhyaksha and Stevenson 
paper that $H(t)$ and $\phi(t)$ arise from the random locations and strengths of scattering elements within the illuminated spot and "the observed [Doppler] signal is therefore a summation of contributions from all these scattering elements". The familiar Doppler signal appearance is shown in figure 2. With reference to signal modulation by laser speckle, it is concluded further into the Rajadhyaksha and Stevenson paper that "speckle effects will ordinarily be negligible in applications of LDV to surface velocity measurements".

It is certainly true that the summation of contributions from the many scattering elements causes the modulation of the Doppler signal. It is exactly this summation from multiple scattering elements which also produces the speckle patterns observed with such systems. Consequently, it is equally correct to describe the phase and amplitude modulation of the Doppler signal as being due to the transition of speckles, each with their own intensity and phase, across a photodetector. Consideration of the effects in a scattering element model has been given previously [1]. The scattering element model tries to follow the approach used for Laser Doppler Anemometry (LDA) measurements in a seeded flow. This is not appropriate for examination of solid surfaces where discrete "scattering elements" cannot be defined. It is entirely accurate, however, to explain the characteristics of the Doppler signal by consideration of the dynamics of the speckle pattern on the photodetector surface.

All discussions concerning passage of scattering elements through the illuminating beams have their parallel in a description of speckle transitions across a photodetector. For example, equation (6) in the Rajadhyaksha and Stevenson paper describes the term $\phi(t)$ in terms of $N_{p}$ 
equal strength scattering elements at initial locations $X_{k}$ and moving with the target surface velocity $V$ as:

$$
\phi(t)=\tan ^{-1} \frac{\sum_{k=1}^{k=N} \sin \left(\omega_{D} x_{k} / v\right)}{\sum_{k=1}^{k=N} \cos \left(\omega_{D} x_{k} / v\right)}
$$

where $\omega_{\mathfrak{D}}=2 \pi f_{D}$. An equivalent term was originally derived in terms of $K$ speckles summed on a photodetector as [2]:

$$
\phi(t)=\tan ^{-1} \frac{\sum_{k=k(t)}^{k(t)+(k-1)} A_{k}(t) I_{k} \sin \phi_{k}}{\sum_{k=k(t)}^{k(t)+(k-1)} A_{k}(t) I_{k} \cos \phi_{k}}
$$

where $I_{k}$ and $\phi_{k}$ are the intensity and phase of the $k^{\text {th }}$ speckle which has area $A_{k}$ on the photodetector at time $t$. Use of the time dependent counter, $k(t)$, indicates a changing population of speckles on the detector at any time. Only this latter model allows consideration of the integrating effect of photodetector size on the Doppler system noise

Further, it is also possible to establish speckle based arguments for the frequency broadening mechanisms, namely finite transit time broadenings and velocity gradient broadenings, discussed in their paper. As speckles evolve across the photodetector, in response to target motions, the phase and amplitude of the Doppler signal vary according to the speckle summation at any instant in time. 
Finite transit time broadenings, resulting from each scattering element producing a burst of signal only for the time for which it is in the illuminating beam, are equivalent to the broadenings due to translation and/or decorrelation of the speckle pattern on the detector as the pattern evolves in sympathy with the target lateral velocity. This causes the phase and amplitude of the resultant photodetector output to vary with the changing speckle summation. Changes in the Doppler signal arise from both the changing population of speckles on the detector as a result of speckle translation and the fact that individual speckles within the pattern are also boiling.

The presence of a velocity gradient across the illuminating beam is indicative of a surface that is tilting. This tilt causes a resultant translation of speckles across the detector at a velocity that is given by twice the product of target-detector separation and the target velocity gradient at the illuminated point. This velocity gradient is equal to the target angular velocity for circumferential incidence on a rotating target [3]. Consequently, velocity gradient broadenings are equivalent to the broadening due to the population of speckles on the detector changing as the speckle pattern translates by this mechanism.

In speckle terms, both of these broadening mechanisms can be combined as "finite speckle residence time broadenings" and this approach has been presented previously [4]. The residence time is related to the time for which each speckle illuminates the detector active area which, in turn, is related to either the relative size of speckles and detector, for the case of translating speckles, or to the speckle lifetime, for the case of a boiling speckle pattern, or to some combination of these two. 
Considerations of scatterers moving through a finite laser beam and of speckles evolving across a finite photodetector are therefore analogous and it is contradictory and erroneous to state that the signal modulation is due to contributions from multiple scattering elements but "speckle effects [are] negligible".

\section{Increased System Accuracy Through Averaging Over Several Cycles}

The paper concludes that accuracy of the LDV system might be improved by averaging over a large number of Doppler cycles to minimise errors in $\phi(t)$. In experiments such as those where a constant velocity is being measured or where there is some a priori knowledge of the variation of velocity with time, it is acceptable to average over several Doppler cycles. For the common application of LDV systems to vibration measurement, however, it is important to consider the relative frequencies of vibration components and Doppler phase noise components. In accordance with equation (7) of the Rajadhyaksha and Stevenson paper, the instantaneous frequency, $f(t)$, of the Doppler signal may be written:

$f(t)=f_{D}+\frac{1}{2 \pi} \frac{d \phi(t)}{d t}$

For the non-vibrating, rotating target in their paper, the spatial characteristics of the speckle pattern on the detector will change but, importantly, will repeat exactly with each rotation of the target. This means that $\phi(t)$ and, therefore, $(d \phi(t) / d t)$ will be pseudo-random with a characteristic spectrum consisting of approximately equal amplitude peaks at the fundamental target rotation frequency and subsequent harmonics. Such 
a spectrum is shown in figure 3 for a target rotating at $5.1 \mathrm{~Hz}$. Of ten these frequencies are of greatest interest in the measurement of target vibrations and it will not be possible to distinguish this speckle induced pseudo-vibration from the genuine vibration information.

It is, therefore, impossible to overcome this noise problem in vibration measurements by averaging over an increased number of Doppler cycles. Averaging of the phase noise would lead to averaging of the vibration data. Additionally, to consider ensemble averaging over either a number of illuminated regions or over a number of vibration cycles would, in many cases, involve use of the assumption that the target behaviour varies neither with time nor across its spatial extent. The pseudo-vibration effect [2] is a significant problem wherever target surface motions produce periodic speckle motions and these considerations must also be given to target motions such as pure tilt and vibration perpendicular to the illumination as well as rotation.

CONCLUSION

In applying laser Doppler techniques to solid surface measurements, it is assumed that the permanent presence of scattering elements and the consequent generation of a continuous rather than a burst type signal overcome many of the practical problems associated with LDA. This is only partially true in terms of signal amplitude and, additionally, a new problem of Doppler phase noise emerges. The important difference occurs because the seeded fluid contains scatterers free to move relative to one another whereas the solid surface consists of scatterers rigidly fixed to 
one another. The speckle pattern formed by scattering from the solid surface can still cause a low signal amplitude when a dark speckle is collected or when the phasor addition of several speckles produces this output. A second and more significant problem, however, arises because the speckle pattern repeats exactly whenever the same population of scatterers is illuminated. This common occurrence with vibrating surfaces has no analogy in fluid flow measurement and causes the Doppler phase noise to be pseudo-random.

Two consequences of the speckle induced phase noise have been detailed in this letter. The first describes the evaluation of Doppler frequency broadenings in terms of speckle lifetimes and residence times on the photodetector. The second concerns the match between the frequency content of the vibration of interest and the frequency content of the pseudo-random Doppler phase noise and the inability to average out this noise without losing the required vibration data.

It is possible to develop satisfactory frequency broadening arguments by initial consideration of "scattering elements" on a rough surface but any such analysis must include, at some point, the consideration of speckle dynamics and the effect of photodetector size. It is better, therefore, to consider speckle pattern dynamics from the outset since the speckle behaviour has the ultimate influence on the Doppler system performance. 
REFERENCES

[1] C.J.D.Pickering, N.A.Halliwell and T.H.Wilmshurst, "The Laser Vibrometer: A portable Instrument" J. Sound \& Vib. 107(3), pp471-485, 1986.

[2] S.J.Rothberg, J.R.Baker, N.A.Halliwell, "Laser Vibrometry: Pseudo-Vibrations", J. Sound \& Vib. 135(3), pp516-522, 1989.

[3] N.George, "Speckle from Rough, Moving Objects", J. Opt. Soc. Am., 66(11), pp1182-94, 1976.

[4] S.J.Rothberg, N.A.Halliwell, P.G.Eastwood, T.H.Wilmshurst, "The Laser Torsional Vibrometer: Noise-Floor Considerations" Proc .Stress and Vibration - Recent Developments in Industrial Measurement and Analysis, London, March 1989. 
FIGURES

Figure 1: Optical System for the Laser Doppler Velocimeter

Figure 2: Typical Doppler Signal

Figure 3: LDV Output Spectrum Showing Pseudo-Vibration Phenomenon 


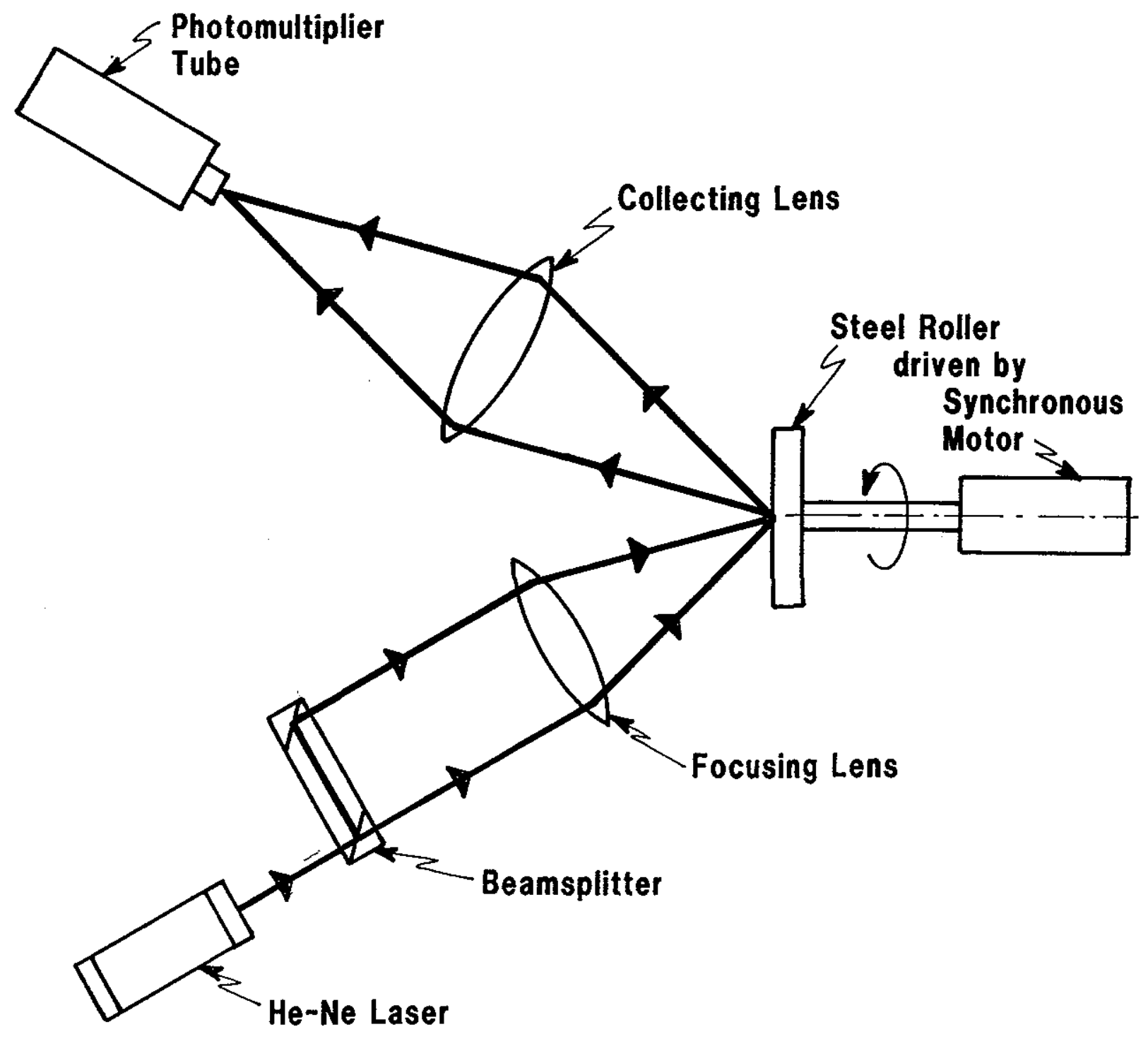


5-May-93

16:09:15

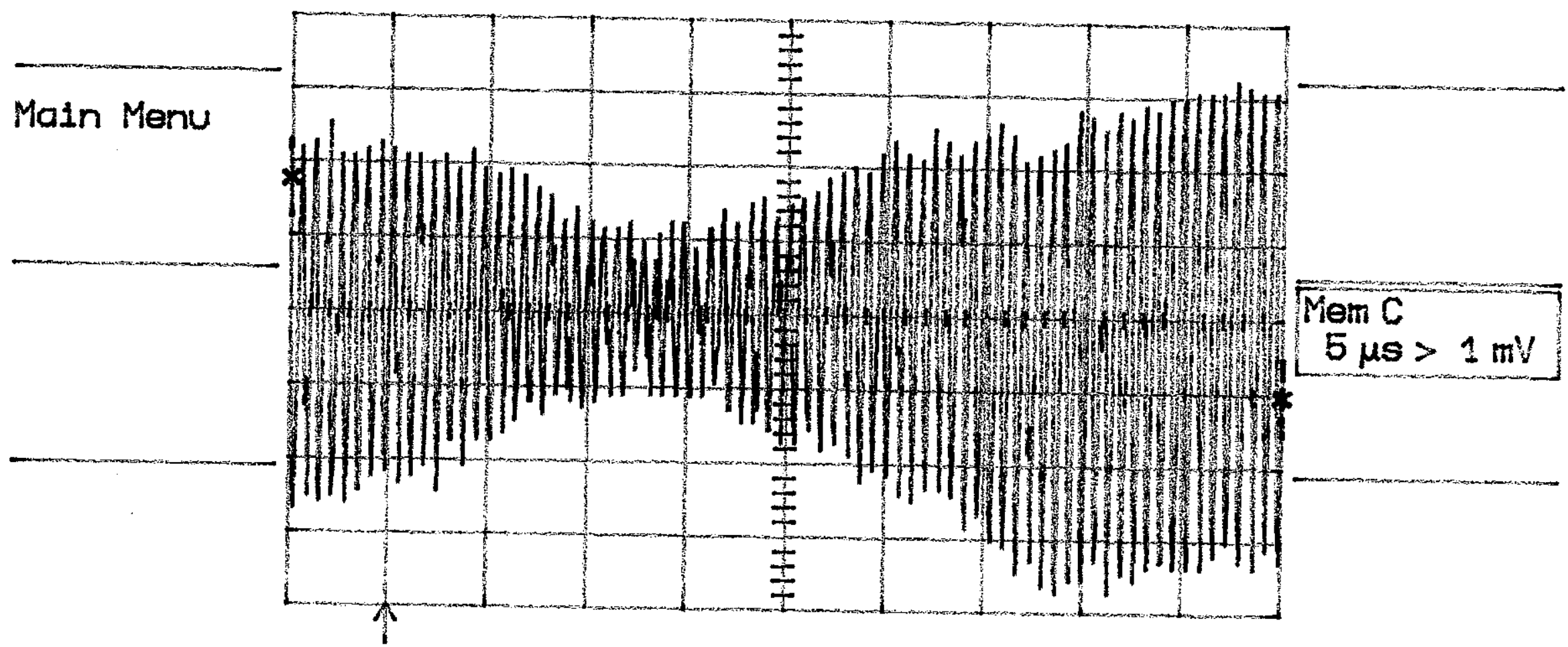

\section{Parameters} Source PASS / FAIL mode
Memory C [ $-5.000 \mu s, 45.000 \mu s] 5000$ pts maximum minimum mean sdev

rms $3.53 \mathrm{mV}$ $-8.97 \mathrm{mV}$ $-2.471 \mathrm{my}$ $2.649 \mathrm{mV}$ $3.622 \mathrm{mV}$ period $=-\overline{-}$
width $=-\overline{-}$
rise
fali $=-\overline{-}$
delay $=--$

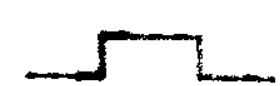

CH1 $5 \mathrm{mV}$ i T/div $5 \mu \mathrm{s}$ 


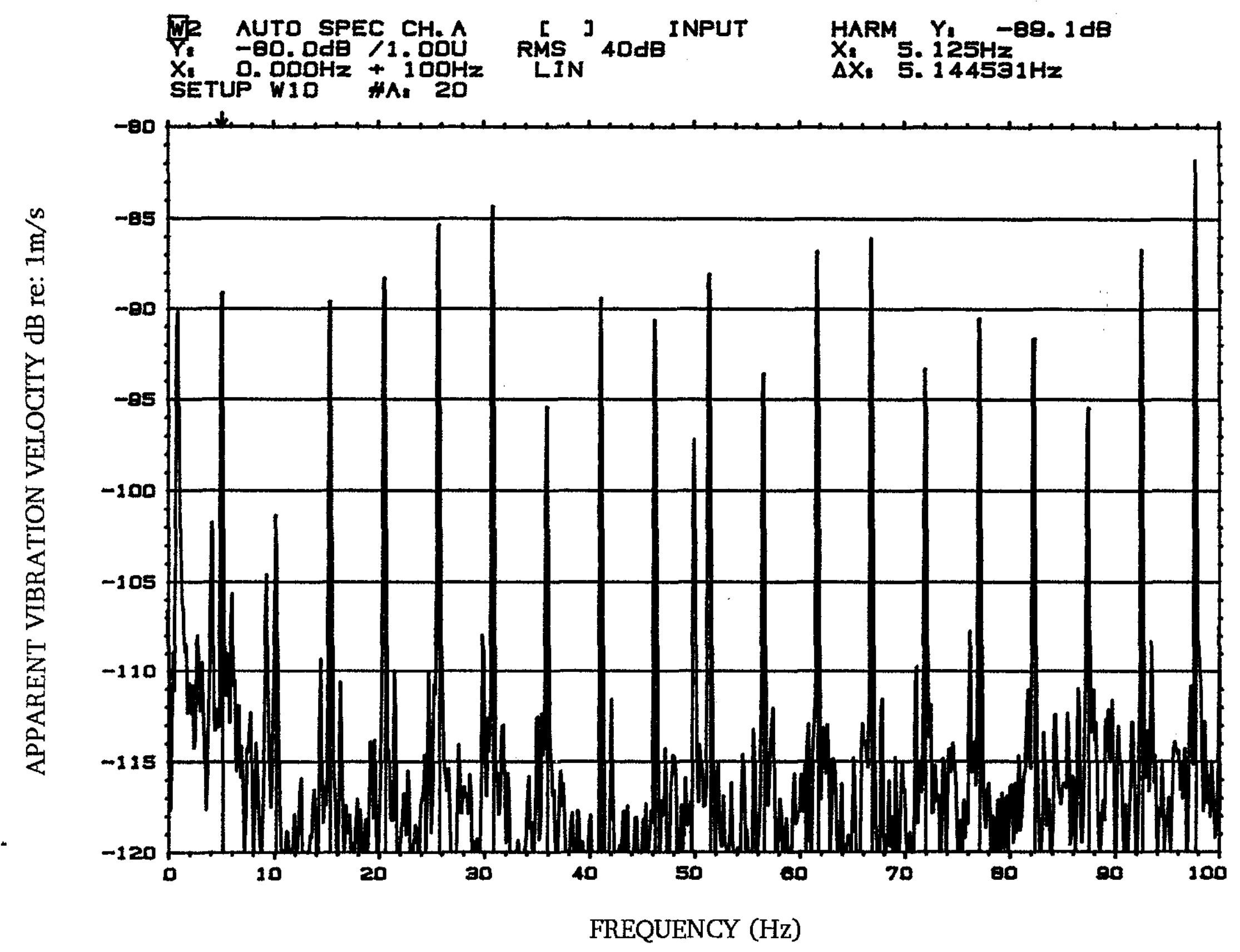

